\title{
Accurate magnetic field measurements of Vega-like stars and Herbig Ae/Be stars ${ }^{\star}$
}

\author{
S. Hubrig ${ }^{1}$, R. V. Yudin ${ }^{2,3}$, M. Schöller ${ }^{1}$, and M. A. Pogodin ${ }^{2,3}$ \\ 1 European Southern Observatory, Casilla 19001, Santiago 19, Chile \\ e-mail: shubrig@eso.org \\ 2 Central Astronomical Observatory of the Russian Academy of Sciences at Pulkovo, 196140 Saint-Petersburg, Russia \\ ${ }^{3}$ Isaac Newton Institute of Chile, St.-Petersburg Branch, Russia
}

Received 8 July 2005 /Accepted 19 September 2005

\section{ABSTRACT}

\begin{abstract}
We obtained accurate circular spectropolarimetric observations of a sample of Vega-like and Herbig Ae/Be stars with FORS 1 at the VLT in an attempt to detect their magnetic fields. No magnetic field could be diagnosed in any Vega-like star. The most accurate determination of a magnetic field, at $2.6 \sigma$ level, was performed for the Vega-like star $\iota$ Cen, for which we measured $\left\langle B_{z}\right\rangle=-77 \pm 30 \mathrm{G}$. In the prototype of Vega-like stars, the star $\beta$ Pictoris, which shows conspicuous signs of chromospheric activity, a longitudinal magnetic field is measured only at $\sim 1.5 \sigma$ level. We diagnosed a longitudinal magnetic field for the first time at a level higher than $3 \sigma$ for the two Herbig Ae stars HD 31648 and HD 144432 and confirm the existence of a previously detected magnetic field in a third Herbig Ae star, HD 139614. Finally, we discuss the discovery of distinctive Zeeman features in the unusual Herbig Ae star HD 190073, where the Ca II doublet displays several components in both $\mathrm{H}$ and $\mathrm{K}$ lines. From the measurement of circular polarization in all Balmer lines from $\mathrm{H}_{\beta}$ to $\mathrm{H}_{8}$, we obtain $\left\langle B_{z}\right\rangle=+26 \pm 34 \mathrm{G}$. However, using only the $\mathrm{Ca}$ II $\mathrm{H}$ and $\mathrm{K}$ lines for the measurement of circular polarization, we are able to diagnose a longitudinal magnetic field at $2.8 \sigma$ level, $\left\langle B_{z}\right\rangle=+84 \pm 30 \mathrm{G}$.
\end{abstract}

Key words. stars: pre-main sequence - stars: magnetic fields - stars: individual: HD 31648 - stars: individual: HD 139614 stars: individual: HD 144432 - stars: individual: HD 190073

\section{Introduction}

Since the discovery of the large infrared excess at wavelengths longward of $12 \mu \mathrm{m}$ from Vega (Aumann et al. 1984), more than one hundred main-sequence stars have been found to have similar infrared excess in the IRAS wavebands and have been called Vega-like stars. They are predominantly spectral type A mainsequence stars with continuum emission at far-infrared wavelengths in excess of that expected from the photospheres of stars of their spectral type. The excess flux is ascribed to thermal emission from orbiting dust grains with a temperature of about $100 \mathrm{~K}$ (Aumann 1985).

One of the prototypes of Vega-like stars, $\beta$ Pictoris, shows evidence of a complex stellar environment with dense and hot regions. It was recently argued that the observed broad emission from the highly ionised species C III and O IV may originate from a solar-like extended chromosphere or from magnetospheric accretion (Deleuil et al. 2001). In the theoretical framework, the disk of this star is truncated at some inner radius, and infalling gas from the disk is channeled by an

* Based on observations obtained at the European Southern Observatory, Paranal, Chile (ESO programme Nos. 072.D-0377, 073.D-0464, 074.C-0463 and 075.D-0507). axisymmetric dipole magnetic field connecting the disk to the star (Hartmann et al. 1994). If solar-like activity is responsible for the observed highly ionised species, a theoretical model to explain the origin and evolution of these phenomena remains to be built. At the moment, the presence of magnetic fields in Vega-like stars has not been convincingly tested whether observationally or theoretically.

Many observational properties (e.g. spectral energy distribution, size and geometrical characteristics of the circumstellar (CS) disk, and, possibly, stellar magnetic field) of young stars depend on the stage of evolution of their CS shells. An evolutionary approach to analysing polarimetric data for Herbig $\mathrm{Ae} / \mathrm{Be}$ and Vega-like stars was recently suggested by Yudin (2000). Herbig Ae/Be stars have spectral types from B to F8 and were first defined as a class in 1960 by Herbig. They show emission lines and display a flux excess in the IR due to the presence of cool and/or hot circumstellar matter (Pérez \& Grady 1997). It was shown that most young stars have a statistically higher value of polarization in comparison to stars that are at more advanced stages of evolution on the main sequence (Yudin 2000). The evolutionary approach can probably also be applied to investigations of magnetic fields in Vega-like stars and Herbig Ae/Be stars. It is very likely that Herbig Ae/Be stars 
are the precursors of most Vega-like stars. The dereddened optical colours of Vega-like stars are consistent with photospheric emission from main-sequence stars. However, for a number of Vega-like stars, the middle/far-IR photometry revealed excess IR emission with colours resembling those of Herbig $\mathrm{Ae} / \mathrm{Be}$ systems (Sylvester et al. 1996; Sheret et al. 2004).

The only attempt to measure a magnetic field in a Vegalike star was done more than twenty years ago by Landstreet (1982). Using a two-channel photoelectric cell polarimeter as a Balmer-line Zeeman analyser, he failed to detect a longitudinal magnetic field in $\beta$ Leo with $1 \sigma$ error bars of $65 \mathrm{G}$.

Although magnetic fields are believed to play a crucial role in controlling accretion onto, and winds from, Herbig $\mathrm{Ae} / \mathrm{Be}$ stars, there is still no observational evidence demonstrating the strength, extent, and geometry of their magnetic fields. There were several attempts in the past to measure magnetic fields in Herbig Ae/Be stars (e.g., Glagolevskij \& Chountonov 2001; Catala et al. 1993), but the first definite evidence of magnetic fields in Herbig $\mathrm{Ae} / \mathrm{Be}$ stars has only recently been presented for the star HD 139614, where a longitudinal magnetic field at $4.8 \sigma$ level, $\left\langle B_{z}\right\rangle=-450 \pm 93 \mathrm{G}$ was diagnosed (Hubrig et al. 2004b). For another Herbig Ae star, HD 104237, a marginal circular polarization signature indicating a longitudinal magnetic field of $\sim 50 \mathrm{G}$ was observed in metal lines by Donati et al. (1997).

On the one hand, the lack of detections of magnetic fields in Herbig $\mathrm{Ae} / \mathrm{Be}$ stars could plausibly be explained by the low accuracy of previous measurements with typical errors up to $1000 \mathrm{G}$ (Glagolevskij \& Chountonov 2001). On the other hand, non-detection of magnetic fields in Vega-like and Herbig $\mathrm{Ae} / \mathrm{Be}$ stars may indicate that large-scale, organised magnetic fields with strengths in excess of a few hundred Gauss are not widespread among these stars. It is obvious that any further attempt to detect fields in these stars must aim at achieving considerably better accuracy and at applying a special observational strategy. The method of Glagolevskij \& Chountonov (2001) allowed them to determine only the average value of the magnetic field from the measurements of a large number of metal spectral lines. However, it is quite possible that, in stars with CS disks, the magnetic field has a complex structure and that strong fields exist only locally, but do so with a topology such that the measurements yield different strength and polarity for different elements and ions. As an example, Babcock (1958) measured a magnetic field of $\sim-270 \mathrm{G}$ on $\mathrm{Ca}$ II $\mathrm{H}$ and $\mathrm{K}$ components, $\sim+270 \mathrm{G}$ for lines of neutral metals, and $\sim 0 \mathrm{G}$ for lines of ionised metals for the Herbig Ae star HD 190073 with a circumstellar disk.

We should note that in our recent study of a few Herbig Ae stars with FORS 1 at the VLT in spectropolarimetric mode (Hubrig et al. 2004b), we also reported the presence of distinctive circular polarization signatures in the Stokes $V$ spectra of the $\mathrm{Ca}$ II $\mathrm{H}$ and $\mathrm{K}$ lines, which appear unresolved at the low spectral resolution achievable with FORS $1(R \sim 2000)$. We also mentioned that, according to a previous study of the Ca II emissivity from dense envelopes near Herbig stars, which used photoionization calculations (Hamann \& Persson 1992), it is very likely that the Ca II lines form very near the photosphere. It is also probable that models involving accretion of circumstellar matter from the disk to the star along a global stellar magnetic field of a specific geometry can account for the Zeeman signatures observed in the Ca II lines. However, the actual geometry of magnetic fields in Herbig Ae stars is completely unknown, and further spectropolarimetric observations at higher resolution in the spectral region around the $\mathrm{Ca}$ II $\mathrm{H}$ and $\mathrm{K}$ lines, as well as other metal lines, could provide an essential clue to the accretion mechanism and the geometry of magnetic fields in these stars.

In this paper, we report an attempt to detect a magnetic field in a small sample of Vega-like and Herbig Ae/Be stars by using circular spectropolarimetric observations obtained with FORS 1 with GRISM $1200 \mathrm{~g}$ at a resolution of $R \sim 4000$, which is twice as high as the one in our previous studies. Our recent magnetic field measurements in the broad hydrogen lines of $\mathrm{A}$ and B-type stars demonstrated that magnetic fields can be measured with an error bar lower than $50 \mathrm{G}$ (Hubrig et al. 2005b). Encouraged by these results we decided to carry out subsequent observations with a non-standard readout mode with a low gain $(\mathrm{A}, 1 \times 1, \mathrm{low})$, which allows the signal-to-noise ratio to be increased by a factor of $\approx 2$. In the following we show that the FORS 1 instrument in spectropolarimetric mode is well suited for accurate magnetic field measurements of the generally broad-lined Vega-like and Herbig Ae/Be stars.

\section{Sample of stars}

The basic data of the Herbig Ae/Be and Vega-like stars studied here are presented in Table 1. The columns indicate, in order, the HD number of the star, another identifier, the visual magnitude, the spectral type, and the stellar parameters $\log T_{\text {eff }}, \log g$, and $v \sin i$. The last four columns list the nearinfrared colour excess $E(V-L)$, the linear polarization $P$, estimates of ages of the stars, and corresponding references. Visual magnitudes, spectral types, and $v \sin i$ values were taken from the SIMBAD database, and the values for the linear polarization and the infrared colour excess $E(V-L)$ were retrieved from Yudin (2000) and references therein. From these data, it is clearly seen that the younger stars have a statistically higher value of polarization and near-IR excess in comparison to stars at a more advanced stage of evolution. One additional normal A-type star, HD 104231, for which previous longitudinal field measurements showed no evidence for the presence of magnetic fields (Shorlin et al. 2002), was selected to check that the instrument is functioning properly and does not introduce any spurious signals. The result of our measurements, $\left\langle B_{z}\right\rangle=+30 \pm 37 \mathrm{G}$, is fully consistent with the previous results of Shorlin et al. (2002) who obtained $\left\langle B_{z}\right\rangle=-93 \pm 53 \mathrm{G}$.

\section{Observations and data reduction}

All longitudinal field determinations for the stars in our sample were obtained from the observations with FORS 1 at the VLT in service mode from April 2003 to June 2005. The multimode instrument FORS 1 is equipped with polarization analyzing optics comprising super-achromatic half-wave and quarterwave phase retarder plates and a Wollaston prism with a beam divergence of $22^{\prime \prime}$ in standard resolution mode. We used the 
Table 1. Basic data of the studied Herbig Ae/Be and Vega-like stars.

\begin{tabular}{|c|c|c|c|c|c|c|c|c|c|c|}
\hline HD & Other & $V$ & Sp. type & $\log T_{\text {eff }}$ & $\log g$ & $v \sin i$ & $E(V-L)$ & $P[\%]$ & Age [Myr] & Ref. \\
\hline \multicolumn{11}{|c|}{ Herbig Ae/Be stars } \\
\hline 31648 & MWC 480 & 7.7 & A3pesh & $3.90-3.95$ & 3.5 & 102 & 2. 61 & $\sim 0.3$ & $2.5-6$ & $1,2,3$ \\
\hline 38238 & V351 Ori & 8.9 & A7IIIe & $3.87-3.89$ & $3.6-3.68$ & $\sim 100$ & $2^{\mathrm{m}}-4^{\mathrm{m}}$ & - & $1-3$ & $4,5,6,7,8$ \\
\hline 139614 & CD-27 10778 & 8.2 & A7Ve & $3.88-3.92$ & $4.0-4.5$ & 13 & $2 \mathrm{~m} 0$ & 0.15 & $5-10$ & $6,9,10$ \\
\hline 144432 & CD-42 10650 & 8.2 & A9Ve & $3.86-3.87$ & $3.9-4.3$ & 54 & 1.94 & $0.1-0.5$ & $1-3$ & $2,9,10,11,12$ \\
\hline 144668 & V856 Sco & 7.0 & A7IVe & 3.89 & $3.5-4.0$ & 204 & $3^{\mathrm{m}}-3^{\mathrm{m}} \cdot 5$ & $0.5-1.3$ & $0.5-1$ & $2,3,6,10$ \\
\hline 163296 & MWC 275 & 6.9 & A1Ve & 3.97 & 4.0 & 133 & 2.96 & $0.5-1.3$ & $3-5$ & $1,2,3,6,10,13$ \\
\hline 190073 & MWC 325 & 7.8 & A2IIIe & 3.95 & 3.5 & 15 & $2 \cdot 46$ & 0.4 & $3-5$ & $1,6,14$ \\
\hline \multicolumn{11}{|c|}{ Vega-like stars } \\
\hline 26676 & HR 1307 & 6.2 & $\mathrm{~B} 8 \mathrm{Vn}$ & $4.06-4.08$ & 4.0 & 175 & $\sim 0^{\mathrm{m}} 1$ & 0.59 & $\sim 70$ & 15,16 \\
\hline 39060 & $\beta \mathrm{Pic}$ & 3.9 & $\mathrm{~A} 5 \mathrm{~V}$ & $3.91-3.96$ & 4.35 & 130 & $0^{\mathrm{m}} 01$ & $\sim 0.02$ & $20-280$ & $17,18,19,20$ \\
\hline 61712 & CD-43 3425 & 9.0 & B7/B8V & - & - & - & $\sim 0.2$ & - & - & \\
\hline 102647 & $\beta$ Leo & 2.1 & $\mathrm{~A} 3 \mathrm{~V}$ & $3.92-3.96$ & 4.30 & 128 & -0.03 & $\sim 0.03$ & $15-330$ & $17,19,20,22$ \\
\hline 109085 & $\eta \mathrm{Crv}$ & 4.3 & $\mathrm{~F} 2 \mathrm{~V}$ & $3.83-3.84$ & 4.26 & 92 & -0.13 & $\sim 0.05$ & $950-1560$ & $11,23,24,25$ \\
\hline 115892 & $\iota$ Cen & 2.7 & $\mathrm{~A} 2 \mathrm{~V}$ & $3.96-3.97$ & - & 90 & $-0 .{ }^{\mathrm{m}} 14$ & $\sim 0.01$ & $\sim 500$ & 25 \\
\hline 219571 & $\gamma$ Tuc & 4.0 & F1III & $3.82-3.88$ & $3.87-3.89$ & 90 & 0.04 & 0.005 & $1700-5720$ & $11,23,24,25$ \\
\hline
\end{tabular}

[1] Pogodin et al. (2005), [2] Acke \& van den Ancker (2004), [3] Natta et al. (1997), [4] Kovalchuk \& Pugach (1997), [5] Hernandez et al. (2005), [6] van den Ancker et al. (1998), [7] Marconi et al. (2000), [8] Balona et al. (2002), [9] Dunkin et al. (1997), [10] Sartori et al. (2003), [11] Nordstroem et al. (2004), [12] Pérez et al. (2004), [13] Swartz et al. (2005), [14] Corporon \& Lagrange (1999), [15] Stickland (1979), [16] Voshchinnikov \& Marchenko (1982), [17] Song et al. (2001), [18] Sheret et al. (2004), [19] Lachaume et al. (1999), [20] Habing et al. (2001), [21] Holmes et al. (2003), [22] Metchev et al. (2004), [23] Ibukiyama \& Arimito (2002), [24] Marsakov \& Shevelev (1995), [25] Wyatt et al. (2005), [26] Greaves \& Wyatt (2003), [27] Lambert \& Reddy (2004).

GRISM $1200 \mathrm{~g}$ to cover the $\mathrm{H}$ Balmer lines from $\mathrm{H}_{\beta}$ to $\mathrm{H}_{8}$, and the narrowest available slit width of $0 . ' 4$ to obtain a spectral resolving power of $R \sim 4000$. For two Herbig Ae/Be stars, HD 38238 and HD 144668, we used the GRISM 600B to cover all $\mathrm{H}$ Balmer lines from $\mathrm{H}_{\beta}$ to the Balmer jump. The spectral resolution of the FORS 1 spectra of these stars is about 2000.

Assessment of the longitudinal magnetic field using the FORS 1 spectra is achieved by measuring the circular polarization of opposite sign induced in the wings of broad lines, such as Balmer lines, by the Zeeman effect. Measurement of circular polarization in magnetically sensitive lines is the most direct means of detecting magnetic fields on stellar surfaces. The errors in the measurements of the polarization were determined from photon counting statistics and converted to errors of field measurements. To obtain a very large signal-to-noise ratio that corresponds to mean longitudinal magnetic field error bars lower than $50 \mathrm{G}$, we took four to eight continuous series of two exposures for each star in our sample using a non-standard readout mode with low gain $(\mathrm{A}, 1 \times 1$,low $)$ and with the retarder waveplate oriented at two different angles, $+45^{\circ}$ and $-45^{\circ}$. In comparison to our previous observations with FORS 1 with the standard readout mode $(\mathrm{A}, 1 \times 1$, high), use of the low gain allowed to increase the signal-to-noise ratio of our spectra by a factor of $\approx 2$. The Zeeman effect increases as $\lambda^{2}$, whereas other line-broadening effects depend linearly on $\lambda$. Hence, as we mainly used the GRISM $1200 \mathrm{~g}$, the error associated with determining the mean longitudinal magnetic field from Balmer lines at longer wavelengths is smaller than when obtained from Balmer lines at shorter wavelengths. The magnetic sensitivity is further enhanced by adding the signal of all individual Balmer lines from $\mathrm{H}_{\beta}$ to $\mathrm{H}_{8}$ that are observed simultaneously with this setup. In the end, the spectropolarimetric capability of the FORS 1 instrument in combination with the large light collecting power of the VLT allowed us to achieve a $S / N$ ratio of up to a few thousand per pixel around $4600 \AA$ in the one-dimensional spectrum. Only for one Vega-like star, HD 26676, is the signalto-noise ratio about a few hundreds due to mediocre weather conditions during the observing night. More details on the observing technique with FORS 1 can be found elsewhere (e.g., Bagnulo et al. 2002; Hubrig et al. 2004a).

\section{Results}

We present in Table 2 our determination of the mean longitudinal magnetic field $\left\langle B_{z}\right\rangle$ for all stars of both samples with the corresponding Modified Julian Dates of the observations. For completeness, our previous measurements of three Herbig Ae stars, HD 139614, HD 144432, and HD 144668, are also included. The mean longitudinal magnetic field is the average over the stellar hemisphere visible at the time of observation of the component of the magnetic field parallel to the line of sight, weighted by the local emergent spectral line intensity. It is diagnosed from the slope of a linear regression of $V / I$ versus the quantity $-g_{\text {eff }} \Delta \lambda_{z} \lambda^{2} \frac{1}{I} \frac{\mathrm{d} I}{\mathrm{~d} \lambda}\left\langle B_{z}\right\rangle+V_{0} / I_{0}$. This procedure is described in detail by Bagnulo et al. (2002) and Hubrig et al. (2004a). Our experience from a study of a large sample of magnetic and non-magnetic Ap and Bp stars revealed that this regression technique is very robust and that detections with $B_{z}>3 \sigma$ result only for stars possessing magnetic fields (Hubrig et al. 2005a).

No magnetic field could be diagnosed in any Vega-like star. The most accurate determination of a magnetic field, at $2.6 \sigma$ 

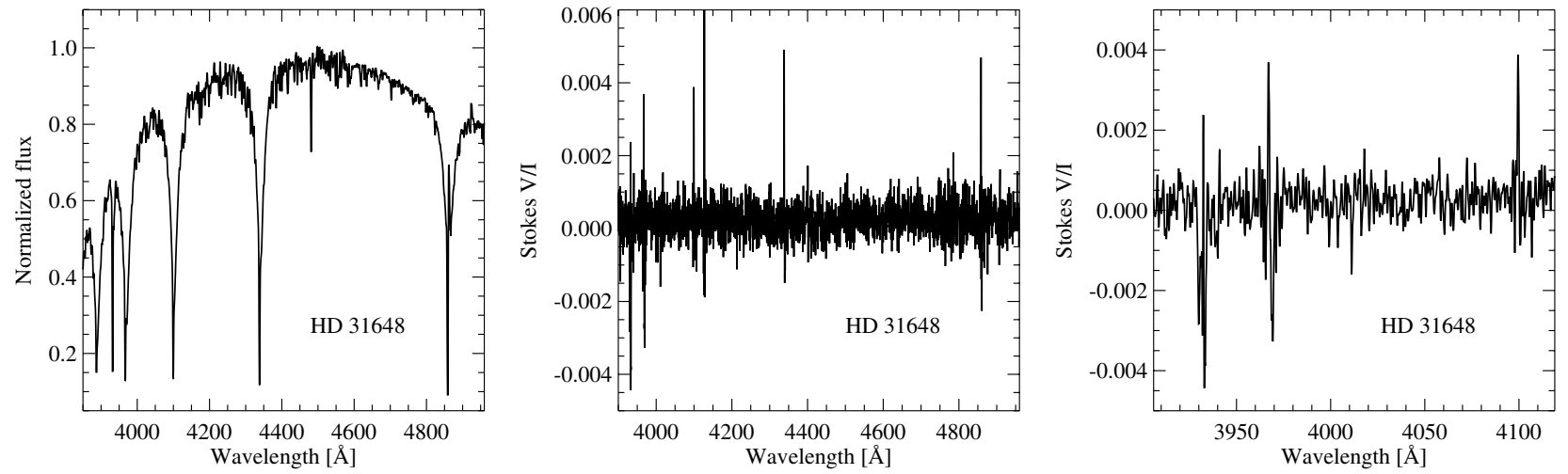

Fig. 1. Stokes spectra of the Herbig Ae star HD 31648: Normalised Stokes I spectrum (left) and Stokes V/I spectrum over the full range (centre) and around the $\mathrm{Ca}$ II doublet and nearby $\mathrm{H}$ Balmer lines (right). Zeeman signatures are clearly seen at the locations of the $\mathrm{Ca}$ II $\mathrm{H}$ and $\mathrm{K}$ and $\mathrm{H}$ Balmer lines.

Table 2. Results of magnetic field measurements of Herbig Ae/Be and Vega-like stars.

\begin{tabular}{|c|c|c|c|}
\hline HD & MJD & $\left\langle B_{z}\right\rangle$ & Remarks \\
\hline \multicolumn{4}{|c|}{ Herbig Ae/Be stars } \\
\hline 31648 & 53296.35 & $+87 \pm 22 \mathrm{G}$ & \\
\hline 38238 & 53249.37 & $-115 \pm 67 \mathrm{G}$ & \\
\hline 139614 & 52904.04 & $-450 \pm 93 \mathrm{G}$ & \\
\hline 139614 & 53405.37 & $-116 \pm 34 \mathrm{G}$ & \\
\hline 144432 & 52900.99 & $-94 \pm 60 \mathrm{G}$ & \\
\hline 144432 & 53447.35 & $-119 \pm 38 \mathrm{G}$ & \\
\hline 144668 & 52901.01 & $-118 \pm 48 \mathrm{G}$ & \\
\hline 144668 & 53120.25 & $-107 \pm 40 \mathrm{G}$ & \\
\hline 163296 & 53279.00 & $-57 \pm 33 \mathrm{G}$ & \\
\hline 190073 & 53519.38 & $+84 \pm 30 \mathrm{G}$ & $\mathrm{Ca}$ II $\mathrm{H}+\mathrm{K}$ \\
\hline \multicolumn{4}{|c|}{ Vega-like stars } \\
\hline 26676 & 53278.33 & $+42 \pm 136 \mathrm{G}$ & \\
\hline 39060 & 53296.26 & $-79 \pm 53 \mathrm{G}$ & \\
\hline 61712 & 53359.25 & $-16 \pm 47 \mathrm{G}$ & \\
\hline 102647 & 53403.37 & $-107 \pm 76 \mathrm{G}$ & \\
\hline 109085 & 53405.32 & $-114 \pm 59 \mathrm{G}$ & \\
\hline 115892 & 53405.34 & $-77 \pm 30 \mathrm{G}$ & \\
\hline 219571 & 53279.03 & $-49 \pm 41 \mathrm{G}$ & \\
\hline \multicolumn{4}{|c|}{ Normal A star } \\
\hline 104321 & 53385.37 & $+30 \pm 37 \mathrm{G}$ & \\
\hline
\end{tabular}

level, has been performed for the Vega-like star $\iota$ Cen, for which we measured $\left\langle B_{z}\right\rangle=-77 \pm 30 \mathrm{G}$. In spite of expecting to find a magnetic field in $\beta$ Pictoris, the prototype of Vega-like stars, which shows conspicuous signs of chromospheric activity (Bouret et al. 2002), a longitudinal magnetic field is measured only at $\sim 1.5 \sigma$ level.

A longitudinal magnetic field at a level higher than $3 \sigma$ was diagnosed for the Herbig Ae stars HD 31648, HD 139614, and HD 144432. The main characteristics of the last two stars, HD 139614 and HD 144432, were already discussed in our previous study (Hubrig et al. 2004b). The pre-main sequence nature of HD 31648 has been studied intensively at different wavelengths during the past years since it was the first Herbig Ae star for which disk rotation was detected (e.g.,
Mannings et al. 1997; Eisner et al. 2004; Vink et al. 2005). Imaging and interferometry studies have found an inclined disk with $i \sim 30^{\circ}$ and PA $\sim 150^{\circ}$. The star is quite young with an age of about 2.5-6.4 million years (van den Ancker et al. 1998). In the FORS 1 low resolution spectra, the strong emission in the $\mathrm{H}_{\beta}$ line is easily identified. The spectrum of this star in integral light is shown in Fig. 1 (left). Stokes $V / I$ spectra of HD 31648 over the whole spectral region and in the spectral region around the Ca II doublet and nearby Balmer lines are presented in Fig. 1 (centre and right). The characteristic Stokes $V$ profiles indicative of the presence of a magnetic field are clearly detected in the $\mathrm{Ca} \mathrm{II} \mathrm{H}$ and $\mathrm{K}$, and Balmer lines.

One Herbig Ae star in our sample, HD 190073, was already measured in the past by Babcock (1958) using Zeeman photographic spectra (see Sect.1). However, the presence of a magnetic field was not confirmed in the later study by Glagolevskij \& Chountonov (2001). This star, frequently called an unusual Ae star, has a very low value of projected rotational velocity $v \sin i$ and displays some atypical characteristics along with properties common to other "classical" Herbig stars. HD 190073 appears to be much more luminous than other Herbig stars of similar spectral type (van den Ancker et al. 1998). Quite recently, Pogodin et al. (2005) studied the temporal behaviour of the $\mathrm{H}$ Balmer lines and the He I $\lambda 5876 \AA$, $\mathrm{NaID}$, and $\mathrm{Ca}$ II $\mathrm{H}$ and $\mathrm{K}$ lines in the spectrum of this star. The most conspicuous spectral feature is the complex structure of the $\mathrm{Ca}$ II $\mathrm{H}$ and $\mathrm{K}$ absorption profiles (Fig 3). It consists of a number of blueshifted absorption components of different width and depth, and it demonstrates a complex variability on timescales from months to decades.

In Fig. 2 (left) we present the low resolution FORS 1 spectrum $(R \sim 4000)$ of HD 190073 in the spectral region $\mathrm{H}_{\beta}$ to $\mathrm{H}_{8}$ to show the extraordinary rich emission line spectrum. It is noteworthy that the Balmer lines are "invisible" in the Stokes V/I spectrum of this star, and no Zeeman signature can be detected at their locations (Fig. 2, centre). On the contrary, distinctive polarization signatures in the $\mathrm{Ca}$ II doublet appear exceptional by displaying several components in both $\mathrm{Ca}$ II $\mathrm{H}$ and $\mathrm{K}$ lines (Fig. 2, right). From the measurement of circular polarization in all Balmer lines from $\mathrm{H}_{\beta}$ to $\mathrm{H}_{8}$, we obtain $\left\langle B_{z}\right\rangle=+26 \pm 34 \mathrm{G}$. However, using both $\mathrm{Ca}$ II $\mathrm{H}$ and 

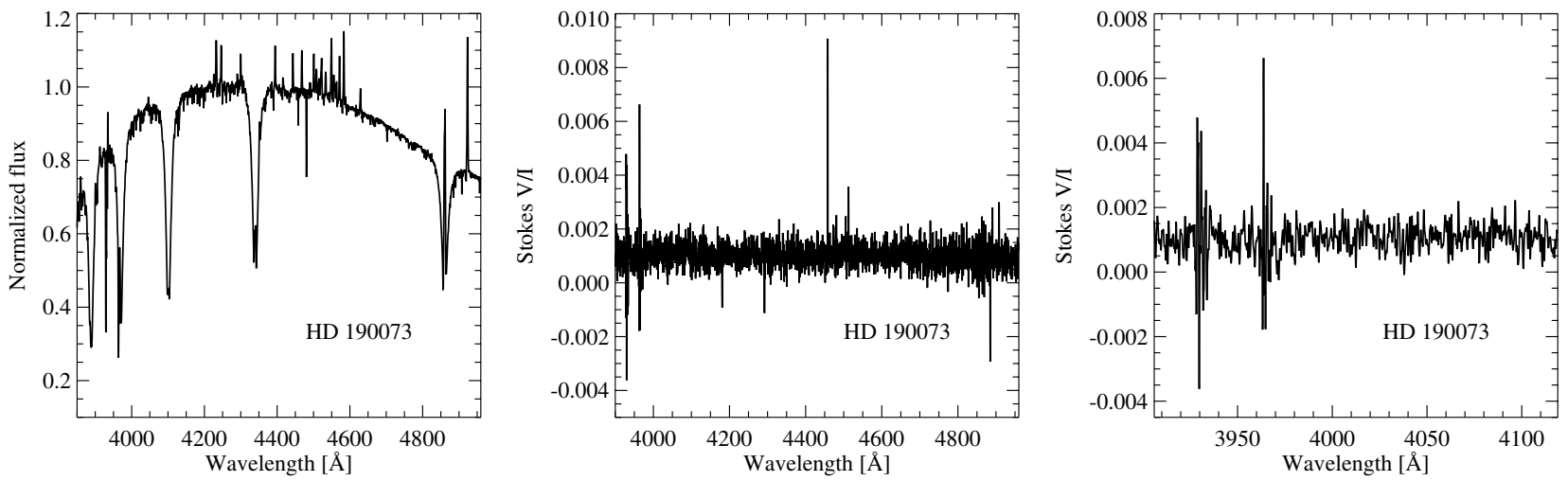

Fig. 2. Stokes spectra of the Herbig Ae star HD 190073: Normalised Stokes I spectrum (left) and Stokes V/I spectrum over the full range (centre) and around the Ca II doublet and nearby $\mathrm{H}$ Balmer lines (right). The spectrum is extremely rich in emission lines demonstrating the presence of a strong stellar wind and a dense circumstellar disk. Zeeman signatures are clearly seen in the Ca II $\mathrm{H}$ and $\mathrm{K}$ lines, but they are not detectable at the locations of the $\mathrm{H}$ Balmer lines.

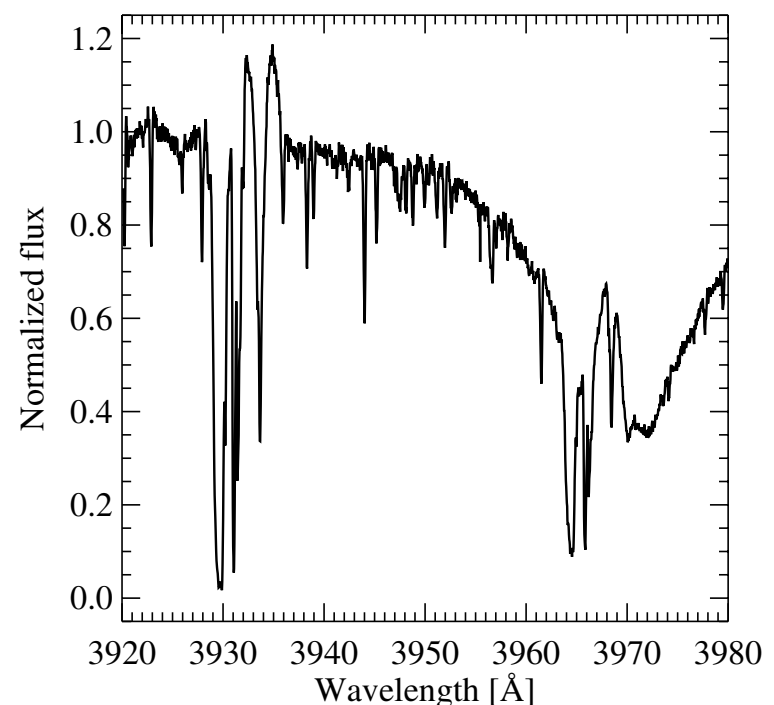

Fig. 3. Observed profiles of $\mathrm{Ca}$ II $\mathrm{H}$ and $\mathrm{K}$ in the high resolution spectrum $(R \sim 48000)$ of HD 190073 using the FEROS spectrograph (Pogodin et al. 2005).

$\mathrm{K}$ lines for the measurement of circular polarization, we are able to diagnose a longitudinal magnetic field at $2.8 \sigma$ level, $\left\langle B_{z}\right\rangle=+84 \pm 30 \mathrm{G}$. This is the value presented in Table 2 .

Both $\mathrm{Ca}$ II $\mathrm{H}$ and $\mathrm{K}$ lines in the Stokes V/I spectrum of HD 31648 display a similar multicomponent complex structure. Since the rotational velocity of this star is rather high $\left(v \sin i=102 \mathrm{~km} \mathrm{~s}^{-1}\right.$ ), complex features in the Ca II H and $\mathrm{K}$ absorption profiles have not yet been reported by other studies of this star in integral light. As mentioned in Sect. 1, it is quite possible that a complex absorption profile structure of the Ca II lines is present in most Herbig Ae/Be stars and might be caused by a specific topology of their magnetic fields. However, due to the fast rotation of these stars, these absorption components are smoothed out and remain undetected.

\section{Discussion}

The presented magnetic field measurements of Vega-like and Herbig $\mathrm{Ae} / \mathrm{Be}$ stars demonstrate that longitudinal magnetic fields in these stars are rather weak. The magnetic field in Vegalike stars, if present at all, is less than $100 \mathrm{G}$, and is almost below the detection limit of spectropolarimetric measurements with FORS 1. A hint of a weak magnetic field is found in the Vega-like star HD 115892, for which the magnetic field is measured at the $\sim 2.6 \sigma$ level. We should note that, as a matter of fact, the detection limit also depends on the structure of the magnetic regions. In the present study we have almost reached the limits of what is currently feasible by using FORS 1 in spectropolarimetric mode.

Confirmation of a magnetic field in the Herbig Ae star HD 139614 and the new detections of the field in two other Herbig Ae stars, HD 31648 and HD 144432, are encouraging and significant, as these detections confirm that the magnetic field does play a role in the star-formation process and that the magnetic accretor scenario, which is rapidly gaining popularity for intermediate-mass pre-main sequence stars, may indeed explain how the PMS stars dissipate their angular momentum, keeping the star rotating as slowly as is observed. However, spectropolarimetric monitoring is required to enable us to probe the strength and geometry of the field responsible for the accretion. Within the magnetic accretor framework, we expect to see changes in polarization properties over the rotational cycle while spots and accretion columns cross the line of sight. Such spectropolarimetric monitoring observations have not been carried out yet. The high accuracy measurements with FORS 1 in spectropolarimetric mode presented here are certainly motivations to ward the next step: a monitoring program of one of the Herbig Ae/Be stars for which the diagnosed strength of the magnetic field is well above the FORS 1 detection limit.

As has been demonstrated for the Herbig Ae star HD 190073 by Babcock (1958), the magnetic field can present different intensity and polarity for different elements and ions. Accordingly, we would like to emphasise the importance of future magnetic field measurements using high resolution spectropolarimeters similar to ESPaDOnS that was recently installed at the CFHT (Manset \& Donati 2003). With such an instrument, it will be possible to measure the magnetic field separately for lines of different elements in order to 
study both the magnetic field configuration in Herbig Ae/Be stars and the interaction of the CS matter with the magnetic field.

Acknowledgements. This research made use of the SIMBAD database, operated at the CDS, Strasbourg, France.

\section{References}

Acke, B., \& van den Ancker, M. E. 2004, A\&A, 426, 151

Aumann, H. H., Beichman, C. A., Gillett, F. C., et al. 1984, ApJ, 278, 23

Aumann, H. H. 1985, PASP, 97, 885

Babcock, H. W. 1958, ApJS, 3, 141

Bagnulo, S., Szeifert, T., Wade, G. A., et al. 2002, A\&A, 389, 191

Balona, L. A., Koen, C., \& van Wyk, F. 2002, MNRAS, 333, 923

Bouret, J.-C., Deleuil, M., Lanz, T., et al. 2002, A\&A, 390, 1049

Catala, C., Bohm, T., Donati, J.-F., \& Semel, M. 1993, A\&A, 278, 187

Corporon, P., \& Lagrange, A.-M. 1999, A\&A, 136, 429

Deleuil, M., Bouret, J.-C., Lecavelier des Etangs, A., et al. 2001, ApJ, 557, L67

Donati, J. F., Semel, M., Carter, B. D., et al. 1997, MNRAS, 291, 658

Dunkin, S. K., Barlow, M. J., \& Ryan, S. G. 1997, MNRAS, 286, 604

Eisner, J. A., Lane, B. F., Hillenbrand, L. A., et al. 2004, ApJ, 613, 1049

Glagolevskij, Yu. V., \& Chountonov, G. A. 2001, BSAO, 51, 88

Greaves, J. S., \& Wyatt, M. C. 2003, MNRAS, 345, 1212

Habing, H. J., Dominik, C., Jourdain de Muizon, M., et al. 2001, A\&A, 365, 545

Hamann, F., \& Persson, S. E. 1992, ApJS, 82, 285

Hartmann, L., Hewett, R., \& Calvet, N. 1994, ApJ, 426, 669

Herbig, G. H. 1960, ApJS, 4, 337

Hernandez, J., Calvet, N., Hartmann, L., et al. 2005, AJ, 129, 856

Holmes, E. M., Butner, H. M., Fajardo-Acosta, S. B., \& Rebull, L. M. 2003, AJ, 125, 3334

Hubrig, S., Szeifert, T., Schöller, M., et al. 2004a, A\&A, 415, 661

Hubrig, S., Szeifert, T., Schöller, M., \& Yudin, R. V. 2004b, A\&A, $428, \mathrm{~L} 1$
Hubrig, S., North, P., \& Szeifert, Th. 2005a, in Astronomical Polarimetry: Current Status and Future Directions, ASP Conf. Ser., in press

Hubrig, S., Szeifert, T., North, P., et al. 2005b, in The Nature and Evolution of disks around hot stars, in press

Ibukiyama, A., \& Arimito, N. 2002, A\&A, 394, 927

Kovalchuk, G. U., \& Pugach, A. F. 1997, A\&A, 325, 1077

Lachaume, R., Dominik, C., Lanz, T., Habing, H. J. 1999, A\&A, 348, 897

Lambert, D. L., \& Reddy, B. E. 2004, MNRAS, 349, 757

Landstreet, J. D. 1982, ApJ, 258, 639

Mannings, V., Koerner, D. W., \& Sargent, A. I. 1997, Nature, 388, 555

Manset, N., \& Donati J.-F. 2003, SPIE, 4843, 425

Marconi, M., Ripepi, V., Alcala, J. M., et al. 2000, A\&A, 355, L35

Marsakov, V. A., \& Shevelev, Yu. G. 1995, BICDS, 47, 13

Metchev, S. A., Hillenbrand, L. A., Meyer, M. R. et al. 2004, ApJ, 600,435

Natta, A., Grinin, V. P., Mannings, V., et al. 1997, ApJ, 491, 885

Nordstroem, B., Mayor, M., Andersen, J., et al. 2004, A\&A, 418, 989

Pérez, M. R., \& Grady, C. A. 1997, SSRv, 82, 407

Pérez, M. R., van den Ancker, M. E., de Winter, D., \& Bopp, B. W. 2004, A\&A, 416, 647

Pogodin, M. A., Franko, G. A. P., \& Lopes, D. F. 2005, A\&A, 438, 239

Sartori, M. J., Lepine, J. D. R., \& Dias, W. S. 2003, A\&A, 404, 913

Sheret, I., Dent, W. R. F., Wyatt, M. C. 2004, MNRAS, 348, 1282

Shorlin, S. L. S., Wade, G. A., Donati, J.-F. et al. 2002, A\&A, 392, 637

Song, I., Caillault, J.-P., Barrado y Navascues, D., \& Stauffer, J. R. 2001, ApJ, 546, 352

Stickland, D.J. 1979, A\&A, 77, 359

Swartz, D. A., Drake, J. J., Elsner, R. F., et al. 2005, ApJ, 628, 811

Sylvester, R. J., Skinner, C. J., Barlow, M. J., \& Mannings, V. 1996, MNRAS, 279, 915

van den Ancker, M. E., de Winter, D., \& Tjin A Djie, H. R. E. 1998, A\&A, 330, 145

Vink, J. S., Drew, J. E., Harries, T. J., et al. 2005, MNRAS, 359, 1049

Voshchinnikov, N. V., \& Marchenko, P. E. 1982, SvA, 26, 673

Wyatt, M. C., Greaves, J. S., Dent, W. R. F., et al. 2005, ApJ, 620, 492 Yudin, R. V. 2000, A\&AS, 144, 285 